\title{
LEIOMYOMA IN THE VESICOVAGINAL SPACE: A CASE REPORT
}

\author{
S.M. RUBINO, P. BARRECA, G. CAMMARATA \\ Department of Obstetrics and Gynecology \\ University of Palermo, Italy
}

Keywords:

Bladder leiomyoma - Paraurethral leiomyoma - Vesicovaginal septum - Vesicovaginal space

\begin{abstract}
The histological features of the leiomyomas located in the vesicovaginal space are similar despite their different anatomic origin. Therefore it is necessary to follow other criteria in order to determine the sites from which they originate. A case of an extravesical pedunculated leiomyoma of the bladder, which had grown in the vesicovaginal space, is reported. Today's imaging studies and surgical findings are of great value in belping to localize the structure from which the tumour has arisen.
\end{abstract}




\section{SUMMARY}

A case of a pedunculated leiomyoma of the bladder, which had grown in the vesicovaginal space, is reported. Criteria for establishing its anatomic origin are highlighted.

\section{INTRODUCTION}

Leiomyomas located in the vesicovaginal space may arise from the bladder, urethra, vagina or from any structure containing smooth muscle. The clinical aspects, pathologic features, and surgical treatment of these uncommon tumours have been previously reported, however the originating sites are not easy to establish. Since there is no histologic difference, some of the cases reported to date do not seem to have ascertained their anatomic origins.

An additional case of this unusual leiomyoma location is reported, and criteria to diagnose its origin are proposed.

\section{CASE REPORT}

A 49 year-old woman presented with an 8-month history of dysuria, dyspareunia and occasional urinary retention. During a routine vaginal examination, a smooth $6 \times 4$ mass was felt through the anterior vaginal wall. The mass was firm, not tender, scarcely movable, and no urethral discharge was obtained from an anterior vaginal wall massage.

A transabdominal and transvaginal ultrasonographic examination showed a leiomyomatous uterus, and an hypoechoic homogeneous mass that displaced the bladder and was connected to the bladder neck by a narrow bridge (fig. 1).

Axial MRI (T1W-T2W) revealed a mass, with a signal similar to that of the uterine fibroids, connected to the bladder neck with a thin pedicle, which lifted an otherwise well defined bladder wall (fig.2).

Cystourethroscopy revealed a laterally displaced urethra, a bladder base elevation, and a normal appear-

Fig. 1 - Longitudinal ultrasonographic image of a solid $4 x 6 \mathrm{~cm}$ mass connected to the bladder.

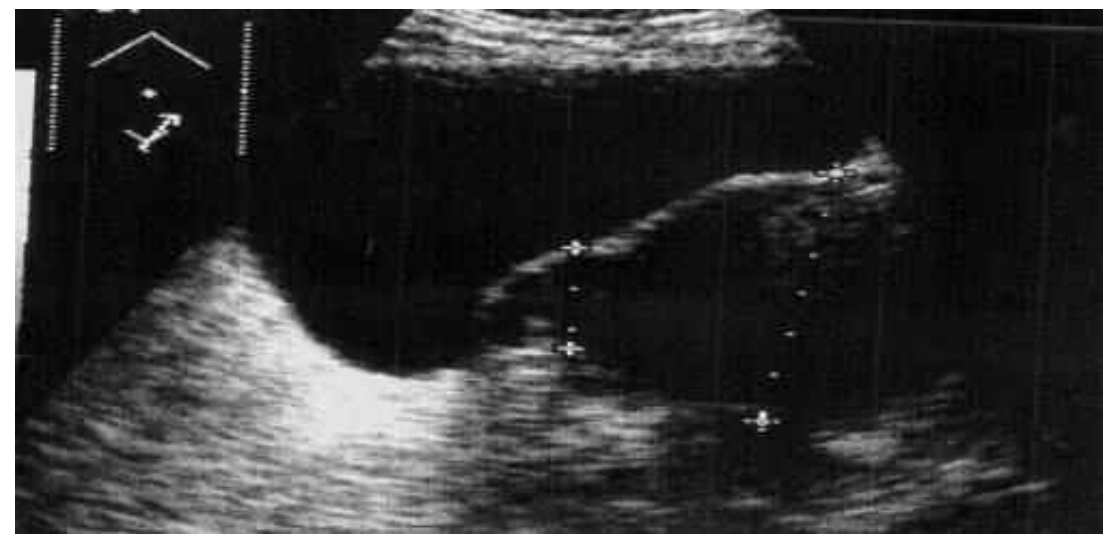


Fig. 2 - MRI sagittal image demonstrates a bomogeneous $4 x 6 \mathrm{~cm}$ mass, isointense with uterus, connected to the bladder wall.

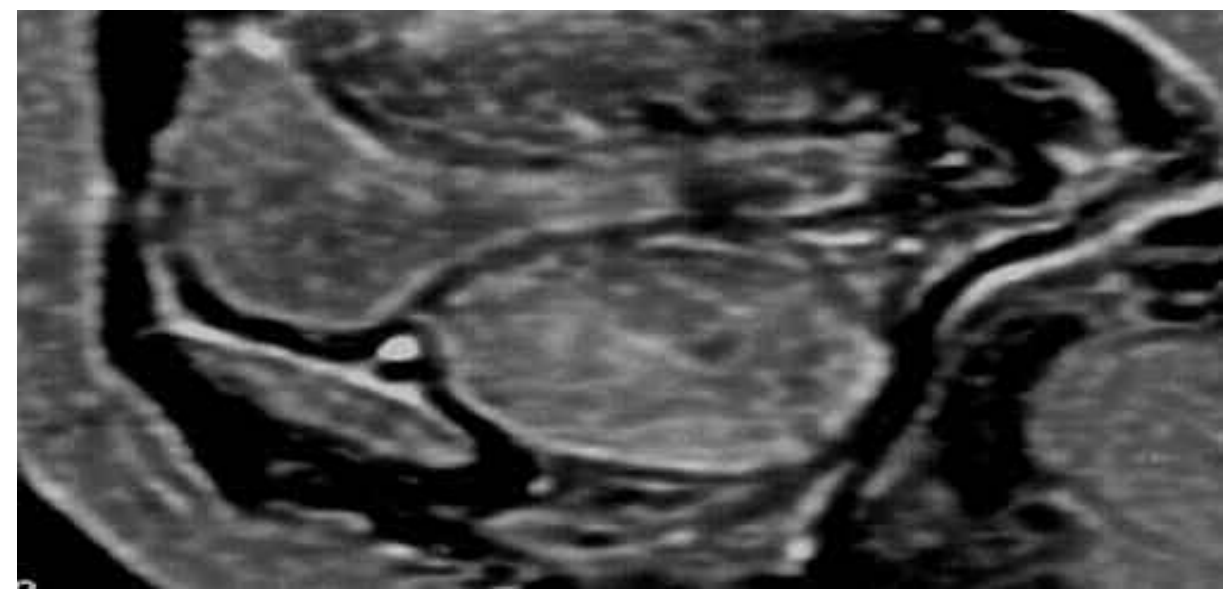

ing urothelium.

The patient was diagnosed as having a pedunculated leiomyoma, which had grown in the vesicovaginal space, possibly originating from the bladder wall. A decision was made for a transvaginal removal.

A $4 \mathrm{~cm}$ longitudinal incision in the anterior vaginal wall was made, and the mass was easily shelled out with a blunt dissection except in proximity of the urethro-vesical junction. A sharp dissection was needed to separate the mass pedicle from the muscular layer of the bladder wall. Several haemostasis ligations were necessary in the pedicle site (fig.3).

Cystoscopy, performed after hav-

Fig. 3 - Gross pathologic specimen of leiomyoma with its pedicle.

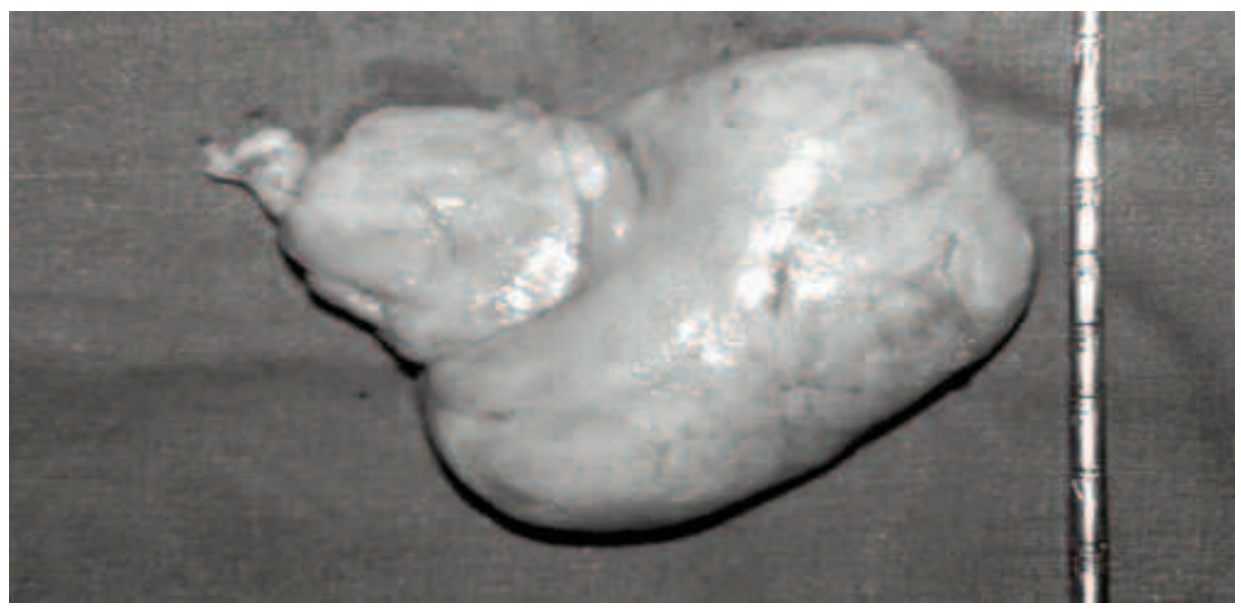


ing removed the mass, showed an intact bladder wall. The endopelvic fascia was repaired and the vaginal wall flaps were approximated. The postoperative course was uneventful. Histology and immunohistochemistry revealed a benign smooth muscle neoplasm.

\section{DISCUSSION}

Although the histological features and the clinical presentations of the leiomyomas growing in the vesicovaginal space are similar despite their different anatomic origins, it would seem that the origin of many of the approximately 350 cases reported to date have only been proven from histopathological examinations. Therefore, it is necessary to follow other criteria in order to determine the different sites from which they originate.

Imaging studies, MR and TVUS, are of great value by characterizing the tumour as well as helping to localize the structure from which the tumour has arisen $(1,2)$.

During surgery, there is major evidence suggesting the anatomic origin of the tumour. First, the site where the mass is so densely intimate that a sharp dissection is required. Secondly, the anatomic structure from which the mass receives its primary blood supply (3).

The origins of some of these uncommon leiomyomas were probably misdiagnosed, not only because most of them were reported prior to today's imaging techniques, but also because the surgical findings were not considered.

Therefore, the incidence of these tumours referring to their different sites of origin should be re-evaluated. The term paraurethral (4) must be reserved to the leiomyomas whose origin has not been defined by the imaging and surgical data.

Correspondence address:

Salvatore M. Rubino, M.D.

Department of Obstetrics and Gynecology University of Palermo, via A. Giordano, 3 Palermo 90127 , Italy Telephone: (091) 6552009

Fax: (091) 6552064

E-mail: profrubino@hotmail.com 


\section{REFERENCES}

1 - RUGGIERI A.M., BRODY J.M., CURHAN R.P. : Vaginal leiomyoma. A case report with imaging findings. J Reprod Med 1966; 41(11): 875-7.

2 - SHADBOLT C.L., COAKLEY F.V., QAYYUM A., DONAT S.M. : MRI of vaginal leiomyomas.

J Comput Assist Tomogr 2001; 25 (3) : 335-7.

3 - CORNELLA J.L., LARSON T.R., LEE R.A., MAGRINA J.F., KAMMERER-DOAK D. :

Leiomyoma of the female urethra and bladder: Report of twenty-three patients and review of the literature.

Am J Obstet Gynecol 1997; 176(6): 1278-1285.

4 - MARTIN J.H., GERRARD E.R. :

Paraurethral Leiomyoma: Does making the distinction make a difference? Infect Urol 2001; 14(2) : 49-52. 